\title{
Color Lesion Boundary Detection Using Live Wire
}

\author{
Artur Chodorowski ${ }^{\mathrm{a}}$, Ulf Mattsson ${ }^{\mathrm{b}}$, Morgan Langille ${ }^{\mathrm{c}}$ and Ghassan Hamarneh ${ }^{\mathrm{c}}$ \\ ${ }^{\mathrm{a} C}$ Chalmers University of Technology, Göteborg, Sweden \\ ${ }^{\mathrm{b}}$ Central Hospital, Karlstad, Sweden \\ ${ }^{\mathrm{c}}$ Simon Fraser University, Burnaby, Canada
}

\begin{abstract}
The boundaries of oral lesions in color images were detected using a live-wire method and compared to expert delineations. Multiple cost terms were analyzed for their inclusion in the final total cost function including color gradient magnitude, color gradient direction, Canny edge detection, and Laplacian zero crossing. The gradient magnitude and direction cost terms were implemented so that they acted directly on the three components of the color image, instead of using a single derived color band. The live-wire program was shown to be considerably more accurate and faster compared to manual segmentations by untrained users.
\end{abstract}

Keywords: segmentation, color, live wire, boundary detection, oral lesions

\section{INTRODUCTION}

The registration of human oral cavity using 3 CCD color camera is today a common practice in odontological clinics. This leads to a huge amount of image data stored in the odontological databases. To efficiently analyze the images we need an automatic segmentation, which can simplify the data analysis and could be used for further feature extraction and computerized diagnostic support system design, in particular to detect potentially precancerous lesions. In this paper we evaluate a semi-automatic segmentation method called live-wire ${ }^{1,2}$, applied to finding the boundaries of the suspicious oral lesions. The live-wire technique can be regarded as a piecewise segmentation method, where the clinician performs the segmentation in smaller steps, at the same time controlling the segmentation accuracy. An important property is that live-wire segmentation can be performed in real-time ${ }^{3}$. These properties give the clinicians a better control over the final result and consequently a better clinical acceptance. The live wire technique can be regarded as a member of active contour models (snakes) family and has been recently incorporated into "united snakes"4. The previous attempts to detect oral lesion boundaries using active contour models can be found in ${ }^{5}$.

\section{MATERIALS AND METHODS}

\subsection{Material}

The image material used in this test was derived from patients treated at the Department of Oral Medicine, Goteborg University and Central Hospital, Karlstad. The dimensions of digitally recorded (3 CCD color camera) images were $768 \times 512$ pixels, RGB values with 8 bits per color channel. The boundaries of the lesions were manually delineated by oral experts, and served as a ground truth in the performance measurement.

\subsection{Color Gradient Extensions}

The local cost function used in the live-wire algorithm can include different terms one of which is usually the gradient information. The gradient image was computed using a vector gradient approach ${ }^{6}$. An image is here defined as a function (a vector field), which maps an n-dimensional (spatial) space to an m-dimensional (color or attribute) space. In our application, the color images map a two-dimensional $(\mathrm{n}=2)$ spatial space $(\mathrm{x}, \mathrm{y})$ into a three-dimensional $(\mathrm{m}=3)$ color space $(\mathrm{u}, \mathrm{v}, \mathrm{w})$. Here $(\mathrm{u}, \mathrm{v}, \mathrm{w})$ represent the corresponding color space coordinates. Let the matrix $\boldsymbol{D}$ represent the derivatives of a vector field: 


$$
\mathbf{D}=\left[\begin{array}{ll}
\partial u / \partial x & \partial u / \partial y \\
\partial v / \partial x & \partial v / \partial y \\
\partial w / \partial x & \partial w / \partial y
\end{array}\right]
$$

Then, the gradient magnitude and the gradient direction are taken as the square root of the largest eigenvalue, $\sqrt{\lambda}_{\max }$, of the matrix $\boldsymbol{D}^{T} \boldsymbol{D}$ and its corresponding eigenvectors, respectively ${ }^{7}$.

\subsection{Cost Terms}

The crucial point of the live-wire algorithm is the construction of its' cost function. Ideally, it should contain all image components that have impact on the position of the lesion boundaries. The live-wire in this work has included the gradient magnitude, gradient direction, Canny edge detection, and Laplacian zero crossing edge detection as part of the cost function. The local cost function $C(p, q)$ from pixel $p$ to a neighboring pixel $q$ is defined as

$$
C(p, q)=w_{Z} f_{Z}(q)+w_{C} f_{C}(q)+w_{G} f_{G}(q)+w_{D} f_{D}(p, q)
$$

where $f_{Z}(q), f_{C}(q), f_{G}(q)$, and $f_{D}(p, q)$ represent the Laplacian zero crossing edge detection, Canny edge detection, gradient magnitude and gradient direction cost terms, respectively. Each cost term is weighted by a corresponding weight constant $w_{Z}, w_{C}, w_{G}$, and $w_{D}$ to allow cost terms to contribute to the total cost at different rates. The local cost term for the gradient magnitude of pixel $q$ is defined as

$$
f_{G}(q)=1-G(q) / \max (G)
$$

where $G(q)$ is the magnitude of the color gradient estimated as $\sqrt{\lambda_{\max }}$ at pixel $q$ and $\max (G)$ is the highest gradient from the entire image. The local cost term is subtracted from 1 so that strong edges give low cost. Similarly, the eigenvector that corresponds to the maximum eigenvalue is inverted for the gradient direction local cost term. The local cost term for the gradient direction from pixel $\mathrm{p}$ going to pixel $\mathrm{q}$ is defined as

$$
f_{D}(p, q)=\operatorname{acos}\left(D_{x}(p) / G(p) * D_{x}(q) / G(q)+D_{y}(p) / G(p) * D_{y}(q) / G(q)\right) / \pi
$$

where $D_{x}(p)$ and $D_{y}(p)$ are the eigenvectors corresponding to the largest eigenvalue for the $\mathrm{x}$ and $\mathrm{y}$ gradient directions of pixel $p$, respectively.

The Laplacian ${ }^{8}$ zero crossing local cost term and the Canny ${ }^{9}$ local cost term were both implemented using the edge detection functions from MATLAB's Image Processing Toolbox ${ }^{10}$. The default settings for the edge detection parameters were used for both edge detectors. The output of these edge detection functions is a binary image with edges represented as pixels containing ones and the rest of the background pixels containing zeros. Therefore, these binary images must also be inverted to give strong edges low costs. Furthermore, the function $C(p, q)$ is scaled by $\sqrt{2}$, if pixel $q$ is a diagonal neighbor to pixel $p$, which corresponds to the Eucledian metric.

\subsection{Live wire operation}

The live-wire program starts by calculating all of the local cost terms except for the gradient direction and then requires the user to choose an initial seed point on the boundary of the lesion. The program then calculates the cost to reach every point on the image, $C(p, q)$, starting from the given seed point by finding the path that produces the minimum cost. This path is likely to be along the edge of the lesion because the cost terms are designed to give edges low costs. The user can then view these minimum paths by pointing to any pixel on the image. The user picks the next seed point based on this visible partial segmentation, which solidifies that partial segmentation. The minimum path cost calculation is then repeated and the process continues until the user is satisfied that the oral lesion has been segmented correctly. 


\section{RESULTS}

The color live-wire segmentation was tested on eight images of oral leukoplakia and lichenoid reactions. For each oral lesion image the manually traced boundary corresponding to the oral expert delineation (Fig.1a) was used to guide the live-wire segmentation (Fig.1b) performed in the RGB color space. Each local cost term was used individually to ensure that it contributed to improving the cost function before adding it to the final cost function. The local cost for the gradient magnitude was calculated using all 3 color components independently instead of taking the average of the RGB components. Using only the gradient magnitude local cost term proved to be a good indicator of the edges surrounding the oral lesion (Fig. 2).
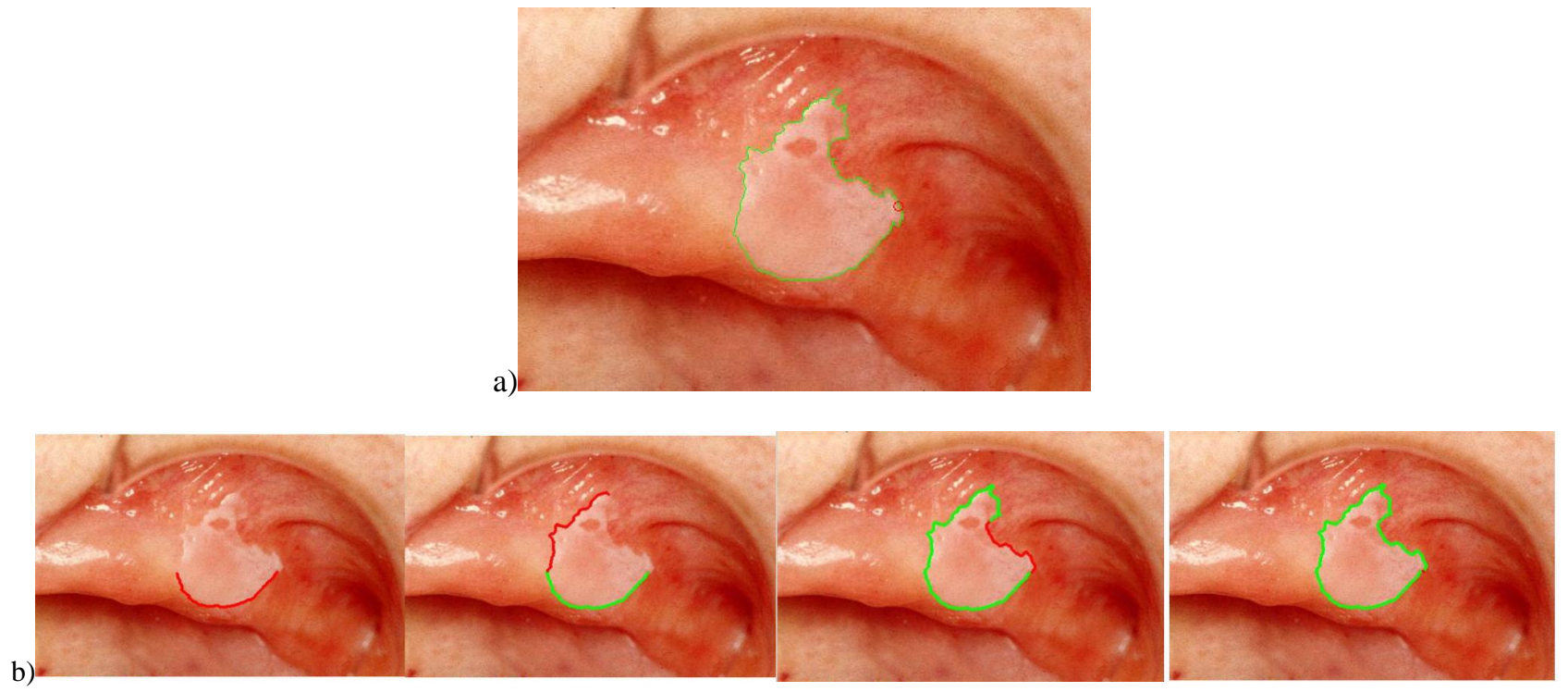

Figure 1. a) "Ground truth" lesion boundary, b) example of piece-wise segmentation using live-wire boundary detection

The segmentations produced by live-wire were evaluated by calculating the distance from all of pixels on the live-wire segmentation to the closest pixel on the expert delineated segmentation. The same distance calculation was performed on manually traced segmentations produced by six untrained users. Using the color gradient magnitude as the only cost function term in the live-wire program, resulted in greater accuracy than the manual segmentations by the untrained users (Fig. 3).

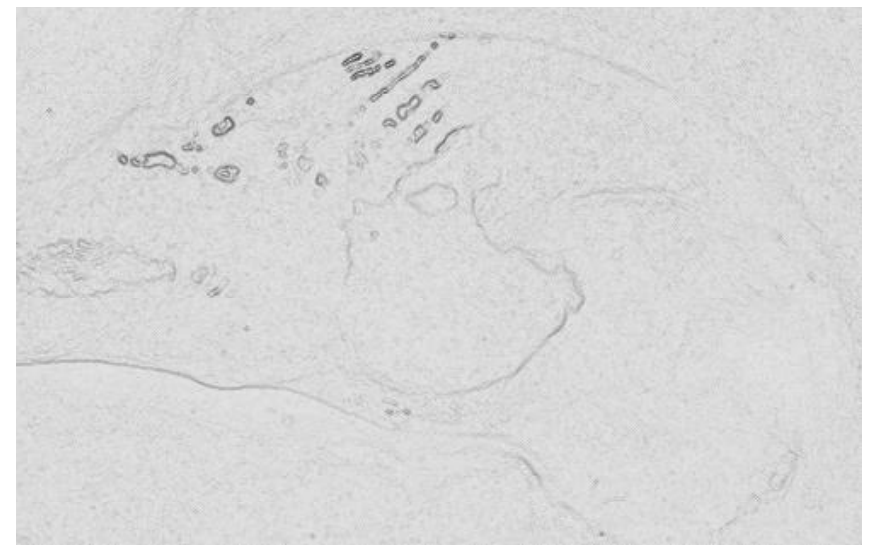

Figure 2. The gradient magnitude of the RGB color image. Note that edges are darker because they are associated with low cost. 


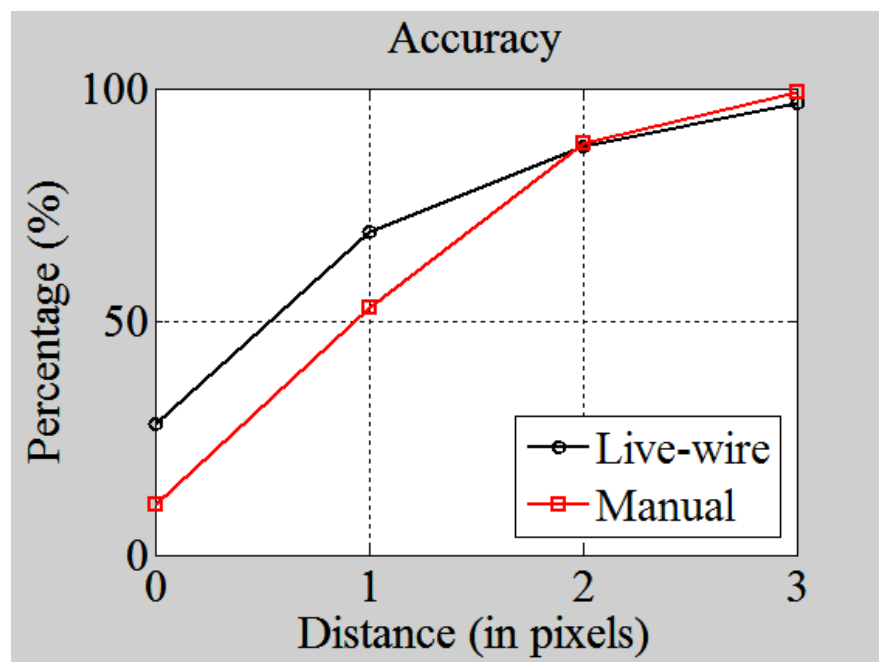

Figure 3. Accuracy comparison between live-wire using only the color gradient magnitude in the cost function and manual segmentations performed by six untrained users. The accuracy is calculated as the percentage of pixels $\leq$ distance from the "expert" segmentation. The manual tracing does slightly better then live-wire at 3 pixels or less, indicating that additional cost terms are needed to improve the live-wire tool.

A comparison between calculating the gradient direction and magnitude using all 3 color components instead of using the averaged color components was performed to determine if there were visible advantages. The live-wire program that operates directly on the three-dimensional color data showed significant improvement over the live-wire program that averaged the three-dimensional color data when compared to the expert segmentation (Fig. 4). This visible improvement supports the use of the cost calculation that uses all three of the color components.

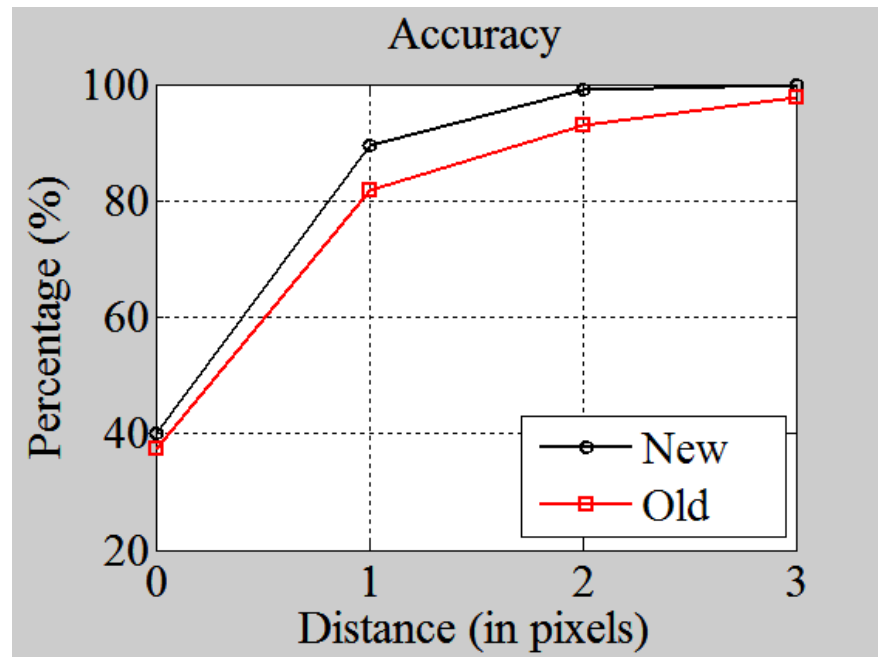

Figure 4. Accuracy comparison between the "New" method that calculates the color gradient magnitude and direction by operating directly on the three dimensional color space compared to the "Old" method that calculates the color gradient magnitude and direction by averaging the color spaces together.

In addition to the gradient magnitude and direction cost terms, Canny edge detection and Laplacian zero crossing cost terms were studied to determine their benefits to the total cost calculation. The Canny method detected in all cases most of the edges that were on the boundary of the oral lesion. However, the Canny method produced many spurious edges 
that could cause the cost calculation to be slightly misguided (Fig. 5a). Nonetheless, these spurious edges may be offset by the other local cost terms in the total cost calculation. The Laplacian zero crossings method did not find as many of the edges on the oral lesion boundary when compared to the Canny method, but less spurious edges were also detected (Fig. 5b). The differences between the two edge detection methods allow them both to contribute to the total cost calculation without having redundant information. The Laplacian zero crossing cost term is weighted slightly more than the Canny cost term because there is a higher probability that the edge actually lies on the oral lesion boundary when using the Laplacian zero crossing edge detection. In other words, the higher weight gives precedence to the Laplacian zero crossing cost term over the Canny cost term when an edge is detected.

The segmented boundaries produced with and without Laplacian zero crossing were compared to the expert delineated boundary. The improved accuracy was barely visible with the addition of the Laplacian zero crossing cost term (Fig. 6); however, the number of seed points entered by the user was reduced when the extra cost term was used. Therefore, the use of both edge detection methods improves the final cost calculation.

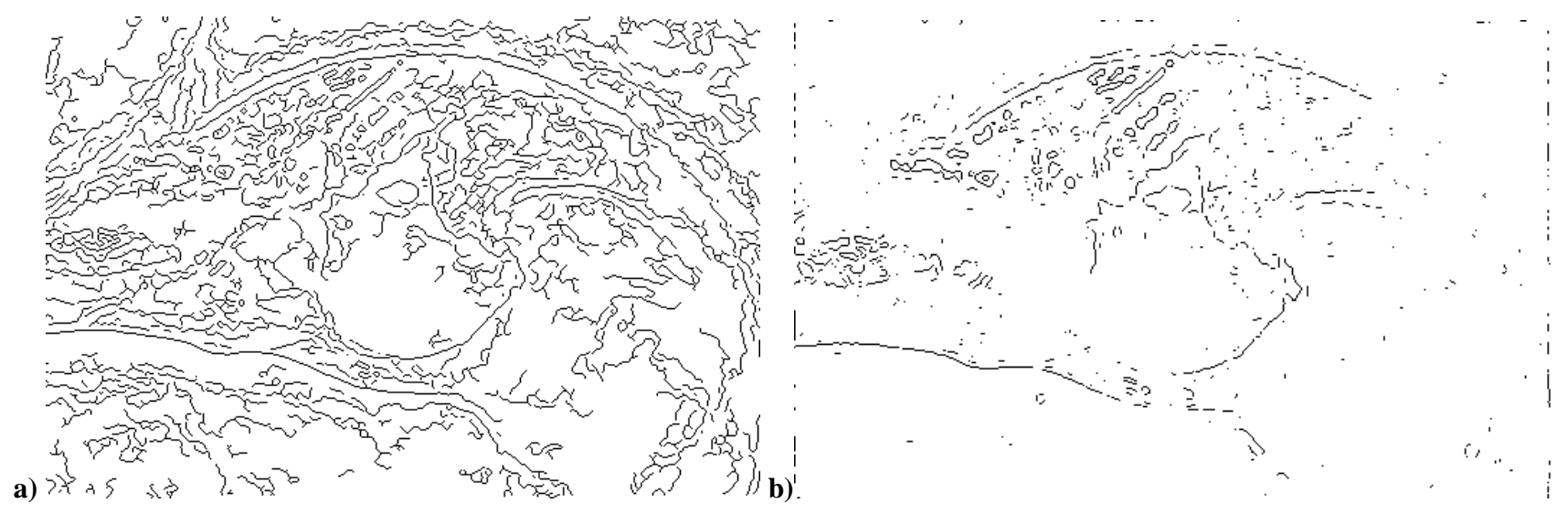

Figure 5. Example of edge detection in an oral lesion image using a) Canny and b) Laplacian zero crossing.

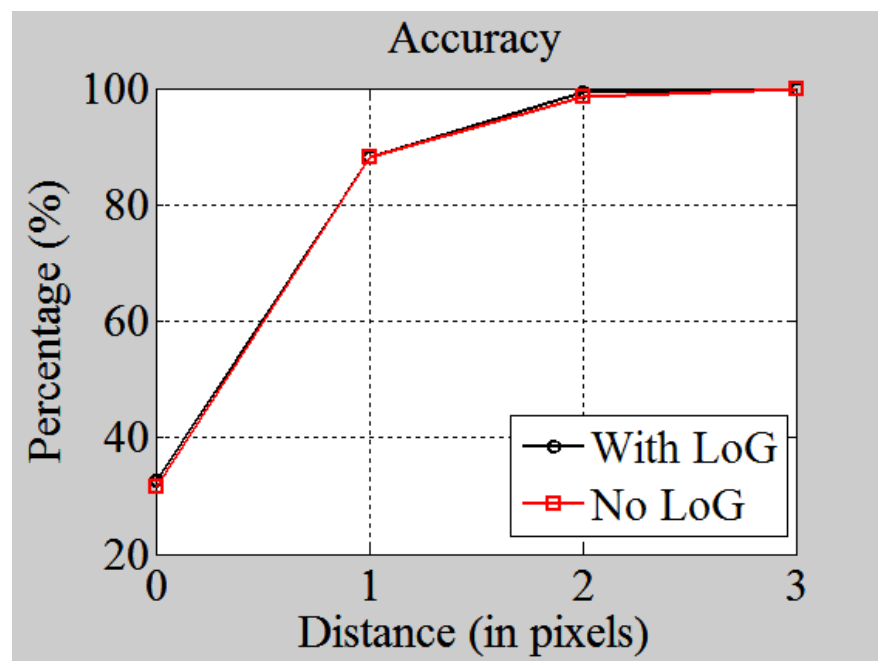

Figure 6. Accuracy comparison between the Live-wire program with and without the Laplacian zero crossing (LoG) cost term added to the total cost calculation. The addition of the extra cost term does not show a major improvement in accuracy, but does reduce the number of user guided seed points needed to produce the segmentation.

The addition of the color gradient direction, Laplacian zero crossing, and the Canny cost terms produced a much more accurate segmentation compared to only using the color gradient magnitude cost term (Fig. 7). The use of these four cost 
terms were used to produce the final cost calculation. The total cost calculation can be visualized for the entire image given a seed point (Fig. 8). The costs increase in a radial fashion around the seed point, but the edges can be seen as darker lines extending away from the seed point. This visualization gives an approximation to the distance that the next segmentation piece can extend to, before needing another seed point.

The application of the live-wire program using all four cost terms provided segmentations with nearly all of the pixels being within 2 pixels and approximately $90 \%$ of the pixels within 1 pixel of the expert delineated segmentation. These segmentations were performed on the cost function with the weights $w_{Z}, w_{C}, w_{G}$, and $w_{D}$ set to $5,3,1$, and 1, respectively. These weights were chosen based on trial and error results and may need to be adjusted for other image sets.

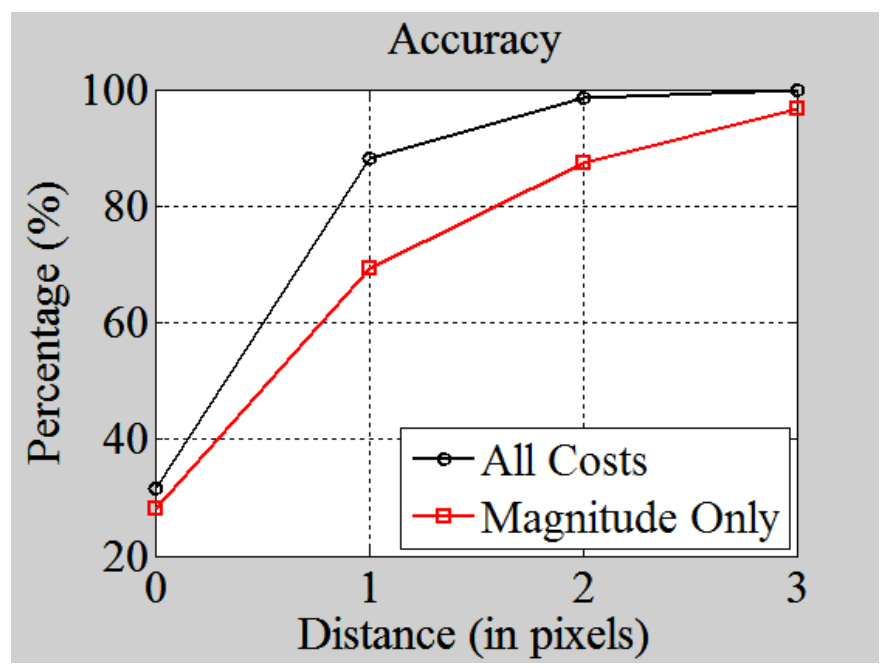

Figure 7. Accuracy comparison between the live-wire program using only gradient magnitude (Magnitude Only) and the live-wire program using gradient magnitude, gradient direction, Laplacian zero crossing, and Canny cost terms (All Costs) compared to expert segmentations.

a)

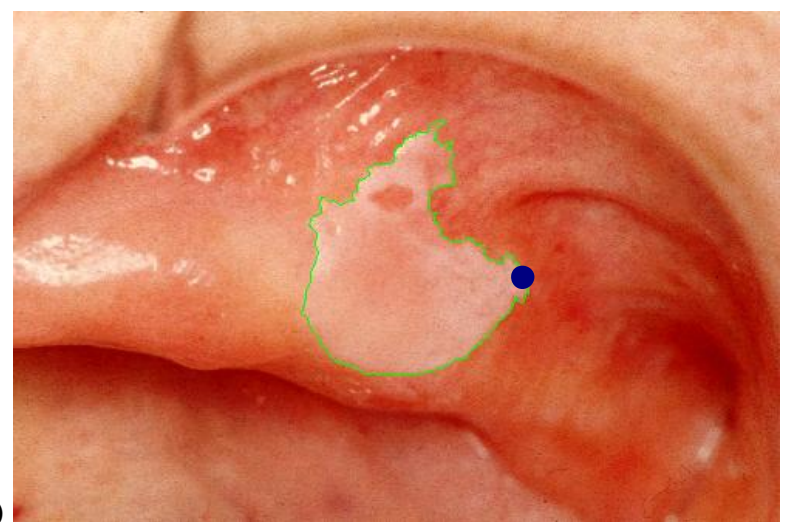

b)

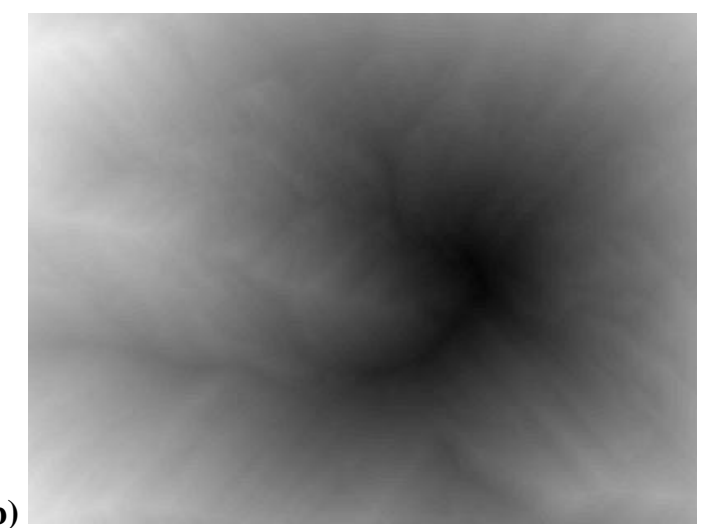

Figure 8. a) Expert delineation of an oral lesion with a given seed point shown as a dot. b) Visualization of the total cost function given the seed point shown in a). Edges of the oral lesion can be seen extending away from the seed point as darker areas that represent pixels with low cost. 


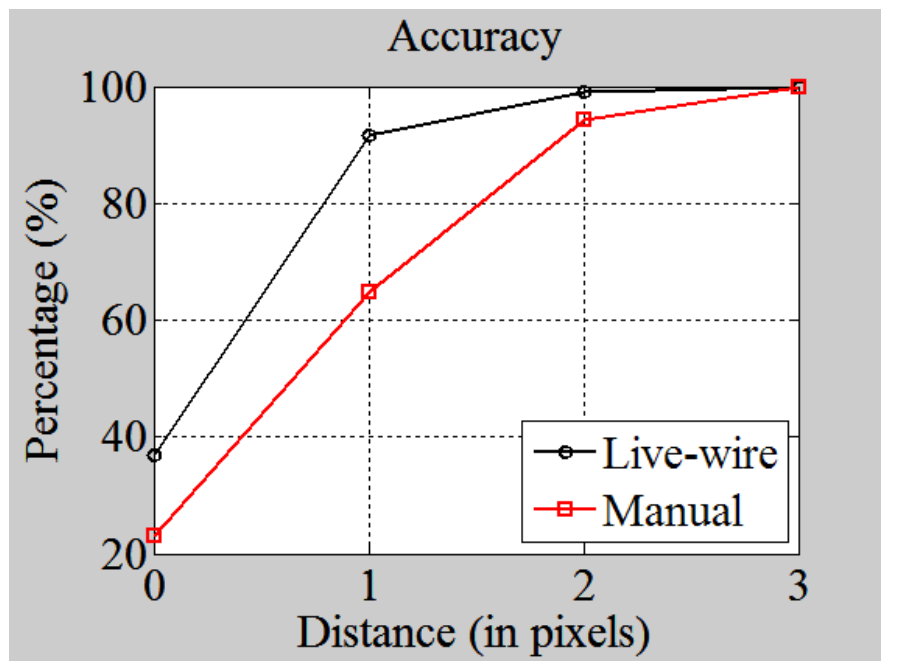

Figure 9. Accuracy comparison between live-wire using all cost terms and the manual segmentations by six untrained users.

\section{CONCLUSIONS}

The presented method makes full use of the advantages of the live-wire segmentation program by allowing experts to control the interactive segmentation process. Using multiple cost terms in the total cost calculation has been shown to produce a more accurate tool for detecting oral lesion boundaries. The tool is further improved by using a method that operates directly on the three-dimensional color data, in contrast to other methods which use only a single derived color band. In this way the color gradient information will not vanish and will efficiently be used for the border detection.

This work contributes to the progress in the field of digital odontology through the development and application of highly-automated image segmentation techniques, which increase the detection accuracy, reduce inter/intra operator variability, and save clinicians time. The developed live-wire color image segmentation simplifies for the clinicians the delineation task of the lesion boundaries and improves the delineation accuracy. It will serve for extracting more effective features for cancerous vs. non-cancerous lesion discrimination.

\section{REFERENCES}

1. W. Barrett and E. Mortensen, "Interactive live-wire boundary extraction,”, Medical Image Analysis 1(4), pp. 331-341, 1997

2. A.X.Falcão, J. K. Udupa, S. Samarasekera, S. Sharma, B. E. Hirsch, R. de Alencar Lotufo, "User-steered image segmentation paradigms: Live wire and live lane", Graphical Models and Image Processing, Vol.60, Issue 4, pp. 233-260, 1998

3. A.X. Falcão, J. K. Udupa and F.Miyazawa, "An ultra-fast user-steered image segmentation paradigm: live-wireon the fly", Proc. of SPIE, Image Processing, Vol. 3661, pp.184-191, 1999

4. J.Liang, T. McInerney and D. Terzopoulos, "Unites Snakes", 7th IEEE International Conference on Computer Vision, pp. 933-940, vol.2, 1999

5. G.Hamarneh, A.Chodorowski and T.Gustavsson, "Active Contour Models: an Application to Oral Lesion Detection in Color Images", Proceedings of IEEE 2000 Conference on Systems, Man and Cybernetics, Nashville, USA, pp. 2458-2463, 2000

6. H-C. Lee and D. R .Cok, "Detecting boundaries in a vector field", IEEE Trans on Signal Processing, Vol.39, No.5, pp. 1181-1194, 1991

7. G. Sapiro, "Vector-Valued Active Contours", Proceedings on Computer Vision and Pattern Recognition (CVPR), pp. 680-86, 1996 
8. E.Hildreth and D.Marr, "Theory of Edge Detection”, Proceedings of Royal Society of London, 207, pp.182-217, 1980

9. J.Canny, "A Computational Approach to Edge Detection", IEEE Transactions on Pattern Analysis and Machine Intelligence, Vol. PAMI-8, No. 6, pp. 679-698, 1986

10. MATLAB Image Processing Toolbox User's Guide, The Mathworks Inc., Natick, Massachusetts, www.mathworks.com , 2004 